\title{
The TOLIMAN space telescope
}

Peter Tuthill, Eduardo Bendek, Olivier Guyon, Anthony Horton, Bryn Jeffries, et al.

Peter Tuthill, Eduardo Bendek, Olivier Guyon, Anthony Horton, Bryn Jeffries, Nemanja Jovanovic, Pete Klupar, Kieran Larkin, Barnaby Norris, Benjamin Pope, Mike Shao, "The TOLIMAN space telescope," Proc. SPIE 10701, Optical and Infrared Interferometry and Imaging VI, 107011J (9 July 2018); doi: 10.1117/12.2313269

Event: SPIE Astronomical Telescopes + Instrumentation, 2018, Austin, Texas, United States 


\title{
The TOLIMAN space telescope
}

\author{
Peter Tuthill ${ }^{\mathrm{a}}$, Eduardo Bendek ${ }^{\mathrm{b}}$, Olivier Guyon ${ }^{\mathrm{c}}$, Anthony Horton ${ }^{\mathrm{d}}$, Bryn Jeffries ${ }^{\mathrm{e}}$, Nemanja \\ Jovanovic $^{\mathrm{f}}$, Pete Klupar ${ }^{\mathrm{g}}$, Kieran Larkin ${ }^{\mathrm{h}}$, Barnaby Norris ${ }^{\mathrm{a}}$, Benjamin Pope ${ }^{\mathrm{i}, \mathrm{j}}$, and Mike Shao ${ }^{\mathrm{K}}$ \\ aSydney Institute for Astronomy, School of Physics, University of Sydney, NSW 2006, \\ Australia \\ bNASA Ames Research Center, MS 245-6, Moffett Field, CA, 94035, USA \\ 'Subaru Telescope, National Observatory of Japan, 650 N. A'ohoku Place, Hilo, HI 96720, USA \\ dAAO, 105 Delhi Rd, North Ryde NSW 2113, Australia \\ eSchool of Information Technologies, University of Sydney, NSW 2006, Australia \\ ${ }^{\mathrm{f}}$ Caltech Optical Observatories, California Institute of Technology, 1200 E. California \\ Boulevard, MC 11-17, Pasadena, CA 91125, USA \\ ${ }^{g}$ Breakthrough Prize Foundation, 901 New York Avenue, NW, Washington, D.C., USA \\ ${ }^{\mathrm{h}}$ Nontrivialzeros Research, 22 Mitchell Street, Putney, NSW 2112, Australia \\ ${ }^{i}$ Center for Cosmology \& Particle Physics, N.Y.U., 726 Broadway, New York 10003, NY, USA \\ ${ }^{j}$ NASA Sagan Fellow \\ kJet Propulsion Laboratory, M/S 321-134 4800 Oak Grove Drive, Pasadena, CA 91109
}

\begin{abstract}
The TOLIMAN space telescope is a low-cost, agile mission concept dedicated to astrometric detection of exoplanets in the near-solar environment, and particularly targeting the Alpha Cen system. Although successful discovery technologies are now populating exoplanetary catalogs into the thousands, contemporary astronomy is still poorly equipped to answer the basic question of whether there are any rocky planets orbiting any particular star system. Toliman will make a first study of stars within $10 \mathrm{PC}$ of the sun by deploying an innovative optical and signal encoding architecture that leverages the most promising technology to deliver data on this critical stellar sample: high precision astrometric monitoring. Here we present results from the Foundational Mission Study, jointly funded by the Breakthrough Prize Foundation and the University of Sydney which has translated innovative underlying design principles into error budgets and potential spacecraft systems designs.
\end{abstract}

Keywords: Astrometry, Exoplanet detection, high precision astronomical measurement

\section{INTRODUCTION}

The astonishing current rate of planetary discovery (approximately one new exoplanet per day at present) in contemporary astrophysics masks a remarkably wide and pervasive observational blind spot. If we wish to know whether any particular star hosts a rocky planet in the Habitable zone, we have only a remote chance of being able to find out. Most of the announced detections are systems where astronomers got a lucky break, most often in the form of the orbital alignment (odds of order 1\%) resulting in a transit across the face of the star with a corresponding measurable dip in the stellar light.

As a consequence, astronomers have very patchy knowledge of the planetary census of stars in our own galactic backyard within 10 parsecs (32 light-years) or so. While missions like Kepler have given information on the statistical incidence of planets in various stellar environments, we still have no data on whether the nearest handful of sun-like stars such as Alpha Centauri, Tau Ceti and Epsilon Eridani actually host a potentiallyhabitable rocky world. Even worse, with present technologies chances of finding answers are slim. Although the technique of spectroscopic detection of Radial Velocities requires no particularly lucky alignment (as for Transits), it has proved very difficult to push the precision to the levels required for Earth-mass objects orbiting

Corresponding author P.T. at E-mail: peter.tuthill@sydney.edu.au

Optical and Infrared Interferometry and Imaging VI, edited by Michelle J. Creech-Eakman,

Peter G. Tuthill, Antoine Mérand, Proc. of SPIE Vol. 10701,107011J · (c) 2018 SPIE

CCC code: $0277-786 \mathrm{X} / 18 / \$ 18 \cdot$ doi: $10.1117 / 12.2313269$ 
at temperate-zone AU scales. The limiting noise floor is usually intrinsic to the star, so that simply improving the instrumentation does not provide an immediate way forward. Finding Earth-analogs in the immediate solar neighborhood is, however, a critical goal at the top rung of science priority for national astronomy roadmaps everywhere.

As catalogs climb into the thousands of confirmed entries, the era of detection is giving way to the new science of exoplanetary characterisation. The critical questions are now to reveal the diverse planetary chemistry, atmospheric composition and physics, surface abundances and potential for a temperate environment that may support life. In this task, our plentiful sample of exoplanets is confronted with a problem: most are at kiloparsec scale distances and quite beyond the reach of follow-up observations such as adaptive optics imaging that has the potential to isolate the faint planetary light from the glare of the host star. On the other hand, planets (if they could be found) around nearby stars would be at ideal scales for this purpose, potentially yielding spectra in which the fingerprints for potential habitability (Water) or even an active biosphere (Oxygen) might be found.

Quite apart from this immediate scientific utility, discovery of nearby temperate-zone planets would mark a signature accomplishment, setting an inspiring challenge for future generations. These are the places to prospect for the signatures of a biosphere with alien life, and further into the future, these provide targets for interstellar probes (e.g. the Breakthrough Starshot initiative ${ }^{1}$ ), humanity's first stepping stones to interstellar travel.

\section{ASTROMETRIC DETECTION}

\subsection{Astrometry for exoplanets: the ugly duckling of indirect detection}

Astrometric detection involves precise measurement of stellar positions, and is widely regarded as the leading technology presently ready to find earth-mass planets in temperate orbits around nearby FGK stars. ${ }^{2}$ As a planet orbits, the star is tugged in a small circle by reflex motion. With accurate enough registration, the tiny displacements imposed on the host star by the gravity of orbiting exoplanets yields a solution for the planet mass and orbit. Unlike competing methods, there are few blind spots, and the signal generated by companions increases with planet-star separation (converse to both RV and Transit methods) making it ideal to probe habitable zones at larger orbital radii.

The opportunity presented by precision astrometric detection is illustrated in Figure 1. Firstly it should be noted that the amplitudes of excursions in Doppler Radial Velocity detection decline very strongly as a function of spectral type. Curves such as this explain the success of campaigns such as the "pale red dot" project in finding potentially habitable-zone companions to M-dwarfs such as Proxima $\mathrm{Cen}^{3}$ and highlight the difficulty in pushing detections towards true Earth-analogs in FGK systems. However it can be seen from Figure 1 that astrometry gains sensitivity in the opposite direction: signals become progressively larger as habitable orbits are pushed out with rising host luminosity. For stellar systems sufficiently close to the Sun, Astrometry and Radial Velocity form a complementary pair able to probe different parts of the stellar main sequence.

It is also worth noting that while the magnitude of the absolute astrometric deflections continues to increase with stellar temperature, the ability to detect objects within the constraints of any set observing campaign does not. In particular, it is also important to take account of the duration of the orbit which rises to be a substantial fraction of a decade for stars of spectral class A. Any campaign observing over a set duration will therefore lose sensitivity to the hot side of Figure 1 resulting in a peaked curve. Furthermore the larger number of orbital cycles probed at the cooler end will shift peak sensitivity towards the cooler end; our expectations for a mission of several years are that sensitivity will be optimised for true Earth-analog type systems (Earth-mass planet around a Sun-like host in a temperate orbit).

Despite its potential promise, astrometric detection for exoplanetary discovery has not yet entered the mainstream. The angular excursions induced by habitable-zone Earth-analog planets are small - of order one microarcsecond even for best-case targets such as Alpha Centuri. Ground based astrometry campaigns at high precision must fight the considerable additional noise process injected by the starlight path through the Earths turbulent atmosphere. Long-baseline optical interferometers have historically delivered precisions better than 100 microarcseconds, with a recent resurgence of interest prompted by ESOs GRAVITY instrument ${ }^{4}$ with accuracy an order of magnitude better (but still not sufficient for Earth-mass planets). Furthermore the nearest stars to 


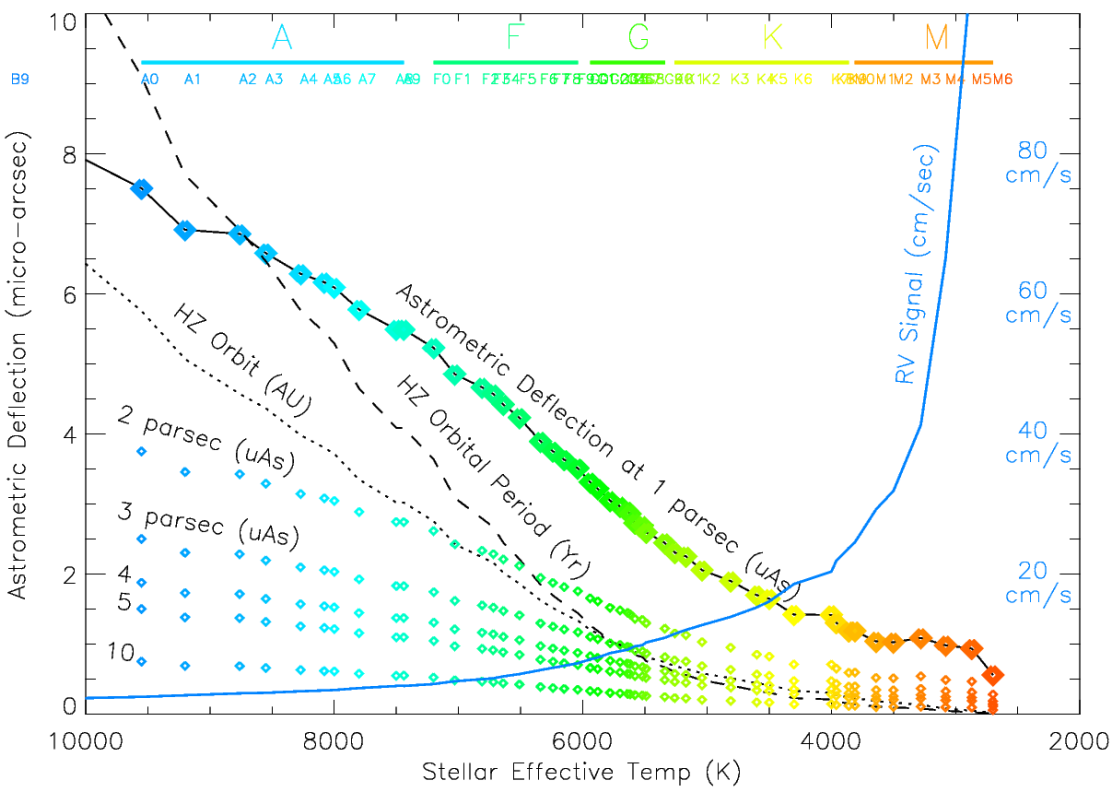

Figure 1. The magnitude of the astrometric deflection (y-axis) imposed by a one Earth-mass planet in a habitable-zone orbit around a main sequence star of a given effective temperature (x-axis). Predictions for hypothetical stars at 1, 2, 3, 4, 5 and 10 Parsec are given (colored diamond symbols). Also overplotted is the corresponding size (dotted line) and period (dashed line) of the "habitable" orbit for which the units of the y-axis must be read in AU and Years respectively. Finally, the solid blue curve rising strongly for M-type stars to the right (to be read against the corresponding y-axis scale to the right) indicates predicted signal strength for a Doppler Radial Velocity measurement.

Earth present a large apparent angular diameter and are correspondingly difficult to observe on long baselines (over-resolved objects have low interferometer fringe contrast).

These intrinsic challenges for ground-based astrometric observation have motivated enduring interest in space. Large space astrometric surveys over wide angles have proved to be extremely productive delivering basic stellar positions, distances and kinematics with the HIPPARCOS mission, and its ambitious successor GAIA which is now measuring a billion stars with precision in the ten(s) of micro-arcseconds. Although this mission is expected to deliver a rich harvest of gas giant planets, pushing detection thresholds down to rocky planets in temperate orbits will require dedicated new technology.

Conventional astrometry measures the position of a star using bright field stars in the neighboring regions of sky as references. This requires field of view as the distance between the science target and sufficiently bright reference stars is many arcminutes or more. Maintaining a stable image scale over large angles is notoriously challenging. Several missions have been proposed by groups in Europe ${ }^{5}$ the $\mathrm{US}^{6}$ and China, ${ }^{7}$ however solving the general problem of micro-arcsecond astrometric detection is challenging.

Viable architectures must find a trade between aperture size and field-of-view. Larger apertures allow more photons to be collected which permits operation with fainter (and therefore closer) background reference stars. However both terms in this trade - large apertures and wide field-of-view - are very considerable drivers of cost and even designs attempting to find the best compromise remain expensive.

\subsection{Narrow-angle Astrometry of Binary Systems}

The fundamental photon noise process motivating this trade is illustrated in Figure 2. Typically our target nearby FGK stars will have magnitudes very much to the left (bright) side of this diagram, a notable example being our prime target Alpha Centuri which has components at about magnitude of 0 and 1.3 mags. For stars as bright as these, the photon-limited signal-to-noise required for an Earth-mass detection is obtained in a matter of only minutes. The astrometric detection is therefore entirely limited by the accumulation of sufficient counts 


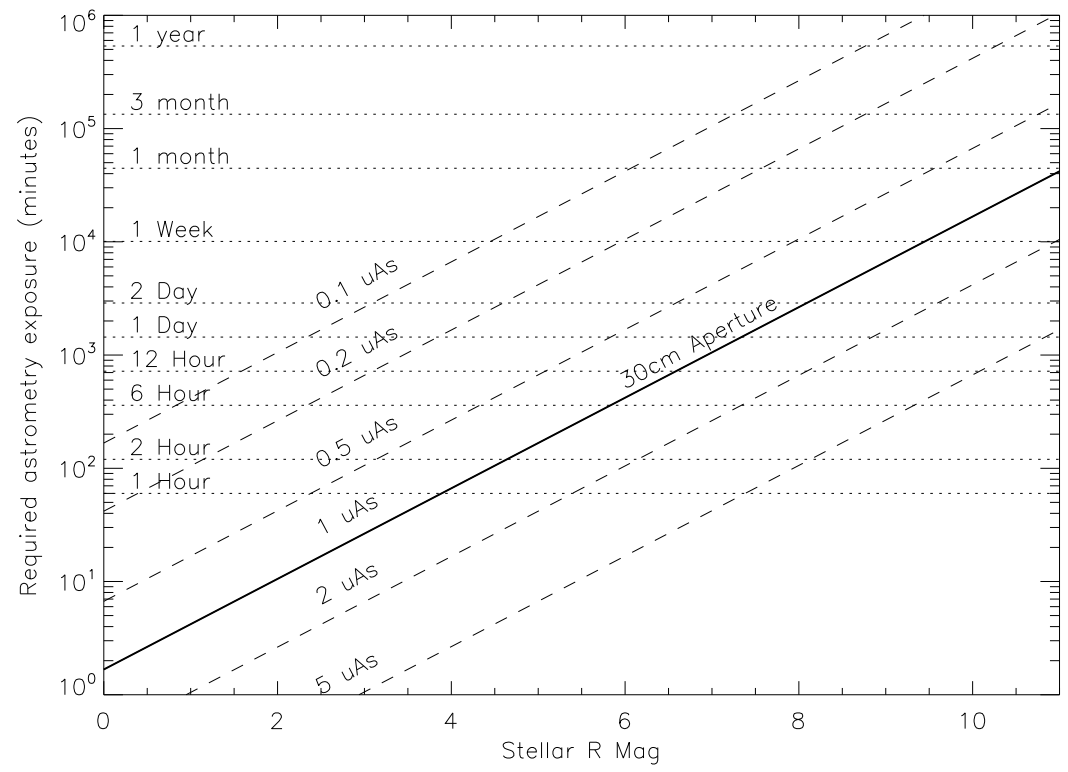

Figure 2. The fundamental detection threshold for an ideal instrument operating at the photon noise limit is given as the integration time required to achieve a given level of astrometric error plotted as a function of the stellar magnitude. The calculations assume an ideal telescope of $0.3 \mathrm{~m}$ aperture operating at $600 \mathrm{~nm}$ and with a $10 \%$ fractional bandwidth.

on the reference stars which, for a field of view of say several arcminutes will (at best) boast a small handful of stars at the extreme right (faint) side of the figure requiring $\sim$ months of integration time. The TOLIMAN* mission was founded on the principle that it is possible to entirely sidestep this dilemma for the special case of observations of binary stars.

In particular, Alpha Centuri itself is almost ideally tailored for a mission exploiting narrow-angle selfreferenced astrometric detection. As our nearest celestial neighbor system, Alpha Cen's solar-analog stars means that habitable-zone exoplanets could be true Earth-twins in year orbits: at the sweet spot for detectability within attainable mission durations and yielding signals factors of 2-10 times stronger than the next-best systems. The two habitable zones have wide enough orbits to yield good signals, yet not so wide as to require an extended mission lifetime for detection. More generally, the key points of advantage and challenge in formulating a mission exploiting narrow-angle binary astrometric detection are:

1. With two bright stars in the field, we immediately circumvent the photon noise problem (minutes to hours exposure with a modest aperture telescope rather than months).

2. The field of view is small: optics can be simple, inexpensive, robust and extremely stable.

3. Most systematic errors in astrometry scale with angle, so that a very nearby reference star gives proportionately better precision than a distant one (for Alpha Cen this gains a factor of $\sim 50$ ).

4. As only the magnitude of the binary separation is measured, the method has blind spots (planets in polar orbits WRT the plane of the binary) and detections do not fully constrain the system (e.g. which star hosts the planet).

5. The method is only suited to multiple star systems within certain bounds of contrast ratio and separation.

6. In removing our reliance on field stars, then the instrument itself must somehow fly an extremely stable element to be used as an astrometric ruler.

${ }^{*}$ Toliman is one of the names given to the Alpha Centuri star system from antiquity. For our mission it also stands for Telescope for Orbital Locus Interferometric Monitoring of our Astronomical Neighborhood 


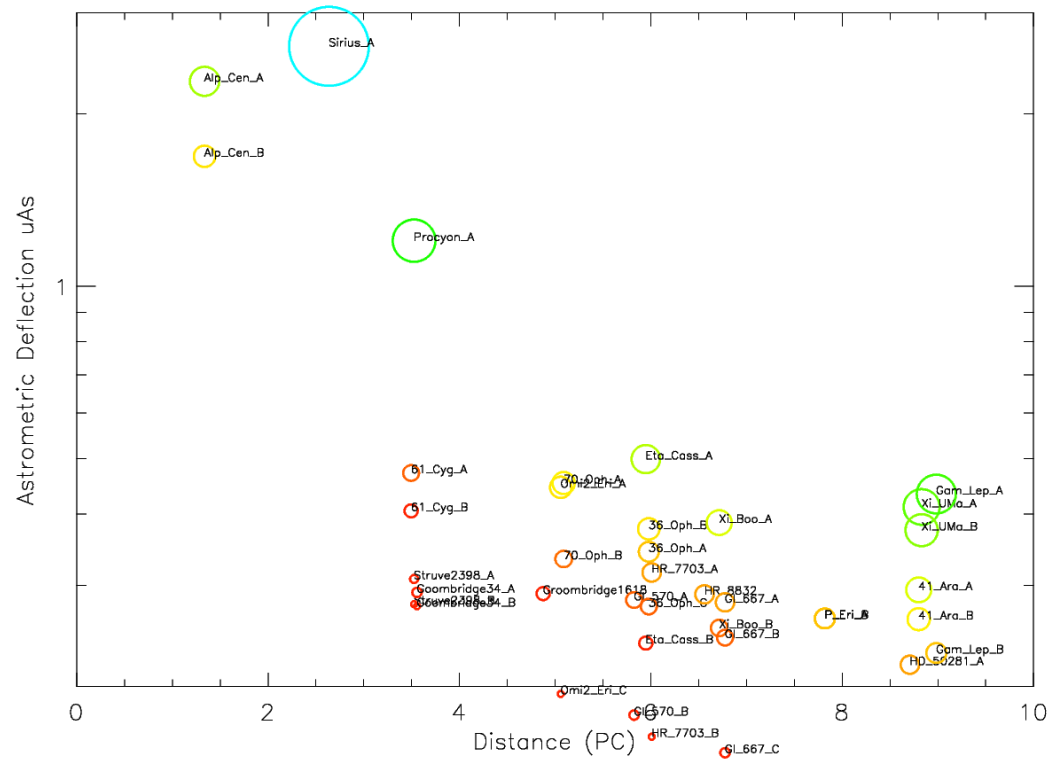

Figure 3. The magnitude of the astrometric signal induced by a temperate-orbit earth-mass planet (y-axis) is plotted for potential targets ordered by distance (out to $10 \mathrm{pc}$ from the sun). Symbol color indicates the spectral class (see Figure 1). Only stars which are in (some kind of) binary configuration are included. Note that most (but not all; a counter-examples being the faint companion objects Sirius B and Procyon B) are suited to the method described herein.

Both the set of pros (1-3) and the set of cons (4-6) listed above constitute strong arguments. The first 3 offer the potential for an agile, inexpensive mission to accomplish significant detections in the most intensely sought-after domain of planetary parameter space. On the other hand, the points $4 \& 5$ emphasize that this is a limited niche that will not provide complete information, and point 6 implies that new technologies will be required to enable it. Taken as a set, these features strongly distinguish this approach from more orthodox field-star-referenced astrometry, placing it in a new and interesting domain.

Although many of the most intensely sought-after targets in the immediate solar neighborhood have no companion and so are not amenable to this technique (prime examples are Epsilon Eridani and Tau Ceti), a plot of all binary stars within $10 \mathrm{pc}$ is given in Figure 3. For completeness, objects such as Sirius and Procyon appear - both have companions and would yield large astrometric signals for a temperate earth-mass planet however neither have configurations suited to our technique. The stars of the Alpha Centuri system are then left as overwhelmingly the most promising targets, although with sufficient sensitivity (a factor of $\sim 5$ better) a handful of potential planet-hosting binaries become available.

\section{THE TOLIMAN SPACE TELESCOPE}

The remainder of this paper is essentially a discussion addressing item 6 in the list above: how can we ensure sufficient measurement stability to record the incredibly small planetary reflex motions without recourse to background field stars in the image plane?

\subsection{Diffractive pupil imaging}

The TOLIMAN mission is empowered by a novel optical architecture which constitutes a reformulation of the idea of a Diffractive Pupil. As originally envisaged, a set of features - most simply a regular array of small opaque dots - are embedded in the pupil of the telescope. ${ }^{8}$ These must be anchored to an element with extreme mechanical stability. The features cause starlight to diffract in the image, essentially forming a pattern whose features are exactly known and stable so long as the diffractive pupil remains stable. 
This innovation offers a cunning solution to the key problem that overwhelmingly dominates astrometric error budgets: the stability of the image. When trying to reference stellar positions at micro-arcsecond scales, a host of small imperfections and minute mechanical drifts, warps and creep of optical surfaces create instabilities that can be orders of magnitude larger than the true signal. Rather than trying to directly contain all these errors, the Diffractive Pupil approach sidesteps them by creating a new ruler of patterned starlight against which to register positions in the image plane. The genius of this approach is that the diffractive grid of starlight suffers identical distortions and aberrations to the signal being measured. Drifts in the optical system therefore cause identical displacements of both the object and the ruler being used to measure it, and so the data are immune to a large class of errors that beset other precision astrometric experiments.

The opaque dots pupil proposed by Guyon ${ }^{8}$ results in a diffraction pattern where the image plane is populated by a regular grid of sidelobe images diffracted from the bright target star. However when considering broadband illumination, bandwidth smearing of the starlight will draw each sidelobe into a narrow radial streak or ray. The signal recovery proceeds by registering the location of these rays against the background field stars. Because the diffractive ruler takes the form of long narrow radial rays, positional information recovered must be in the orthogonal ordinate. Therefore the primary observable consists of the recovery of azimuthal positions of (a rich field of) background stars registered against the nearest diffraction rays.

\subsection{Reformulating diffractive pupils for precision binary astrometry}

For the TOLIMAN mission, the diffractive pupil formulation of Guyon has two fatal flaws: (1) it relies on background field stars and (2) with its radially smeared ruler it is unable to yield precision measurement of the separation of any binary star. For only a single pair of stars, radial information is essential.

Instead, we here propose a novel form of diffractive ruler which generates fine-featured patterns capable of spanning the required separations between the components of a binary star system. Therefore TOLIMAN requires diffractive pupils capable of creating patterns with sharp structure extending in the radial direction. Our primary design driver was to find patterns that create a region on the image plane uniformly filled with features that have the highest gradient energy and that occupy the minimum span in dynamic range. Essentially, the former criteria attempts to optimise our ability to accurately register the resulting pattern - fitting algorithms
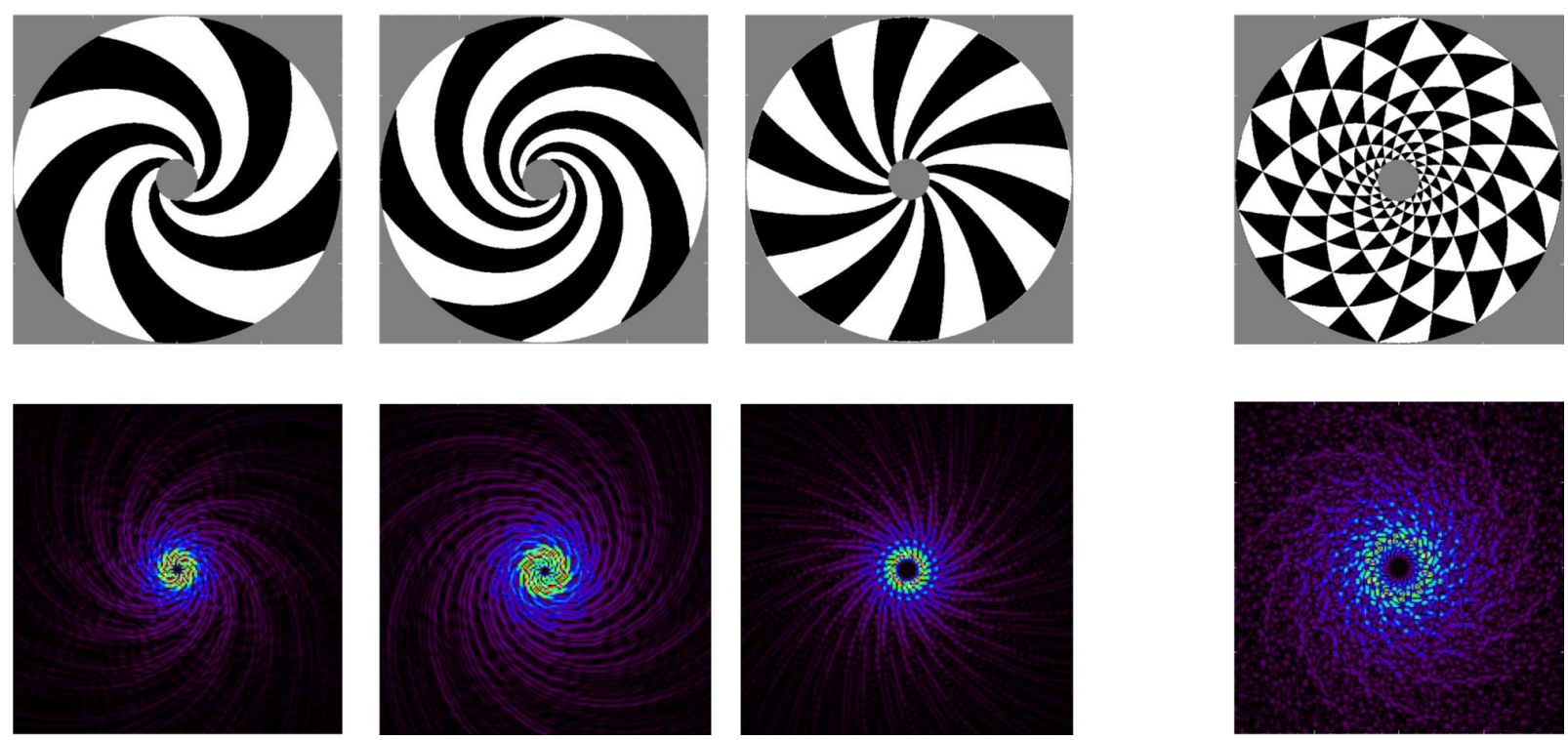

Figure 4. Upper Panels: diffractive phase pupils are arranged with discrete phase steps so that half of the incident starlight (say, white) is assigned a phase of zero while the other (black) is in antiphase at $\pi$ radians. Grey indicates regions with no illumination. Lower Panels: The monochromatic PSF generated by each pupil. The patterns to the left illustrate 3 separate log-harmonic spirals, while to the right is the combined effect of the sum of all 3 log-harmonic spirals (upper) together with the corresponding PSF (lower). 

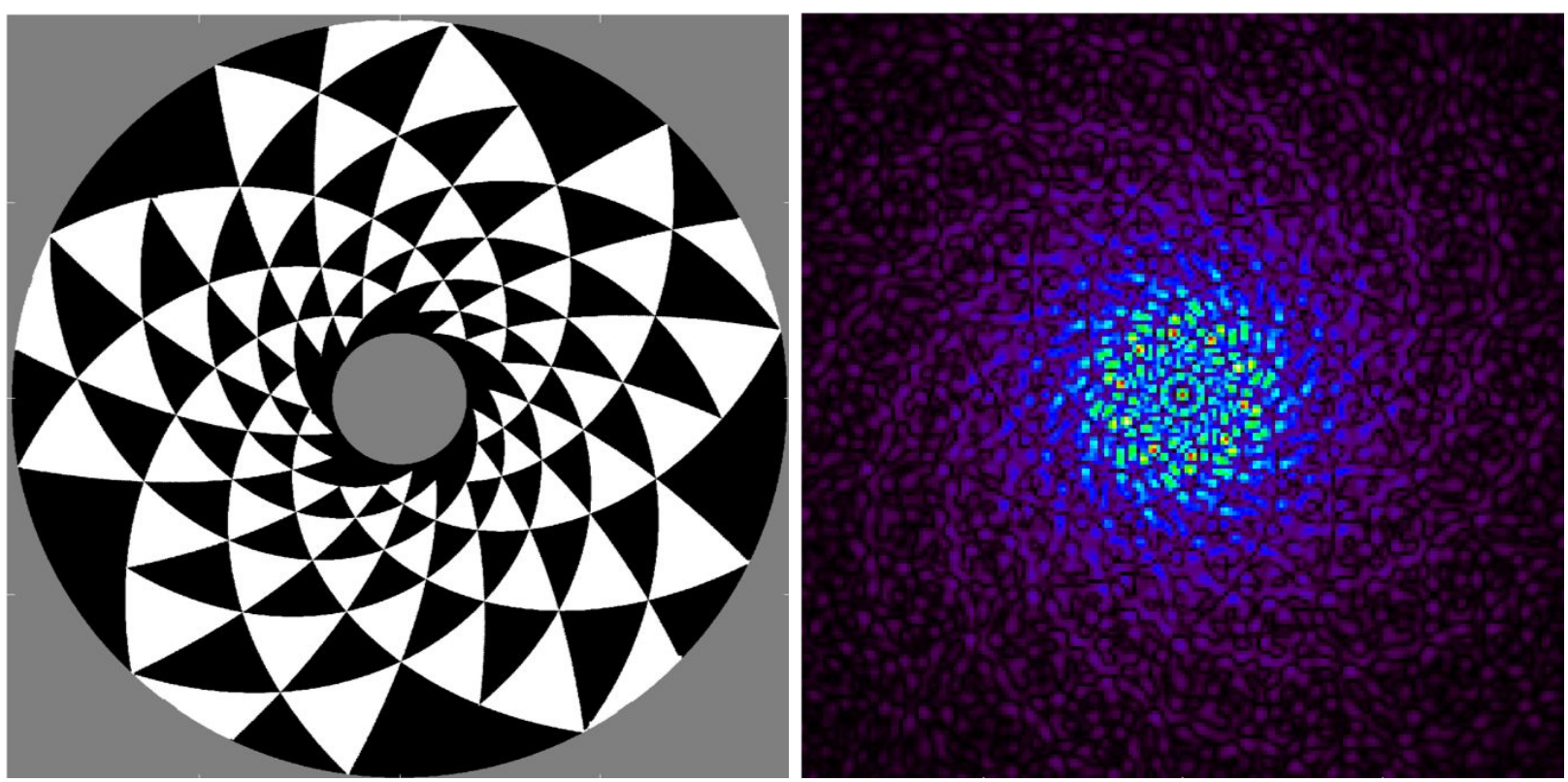

Figure 5. Left Panel: a candidate point-design pupil for the TOLIMAN mission. As for Figure 4, white/black regions indicate discrete phase steps of $0 / \pi$. Right Panel: the monochromatic PSF generated yields a complex and strongly featured pattern extending from the core, uniformly filling the region with sharp fringes.

rely on regions where the image has the strongest slopes or sharp edges. The latter condition is required to spread the starlight preventing saturation of the detector, and spanning the separation of the binary with diffractive features so as to enable the diffractive pupil methodology.

One pathway to optimise a pupil based on these design drivers is given in Figure 4. The pupils depicted exploit strong phase structure with phase steps which divide the incident starlight wavefronts into two interleaved patterns. Placing these two at antiphase has the effect of diffracting all the light away from the PSF core and into an intricate halo of sharp diffractive speckle features. The net result pupil from the figure, consisting of the sum of three log-harmonic spirals (two with 5 -fold symmetry, one with 10-fold symmetry), is seen to exhibit many of the features we are seeking.

However, as noted from Figure 4, the PSF produced from entirely symmetric patterns where there are equal amounts of 0 and $\pi$ phase must (by construction) exhibit a null at the pointing origin. This explains the dark holes to be found at the center of the patterns which are essentially the same as those produced by a phase vortex coronagraph. As we have no requirement for such a central null, and furthermore this is wasteful of pixels over the most favorable region of the PSF, our candidate point-design diffractive pupils were constructed with some minor degree of unbalancing of the $0 / \pi$ symmetry. Such a design is depicted in Figure 5 and is now seen to meet our goal of filling the entire diffractive region, including the core, with sharp structure.

\subsection{Stabilizing the metrology scale}

The point-design pupil of Figure 5 is highly effective in diverting energy into a structured halo of speckles, delivering the previously discussed intrinsic calibration advantages conferred by the Diffractive Pupil. However the global scale of the image-plane diffractive ruler is contingent upon three things: (1) the physical dimensions of the structure diffracting the light, (2) the plate scale of the imaging system and (3) the effective wavelength of the light. While the mission is able to control the first of these to acceptable levels (by way of thermally-stable design), the other two result in an ambiguity. The effective wavelength (3) is subject to variation as the star changes in effective temperature with surface activity, while the plate scale (2) will vary slightly with imperfect stability of the optics. The requirement for micro-arcsecond astrometric precision therefore mandates a way to dis-entangle these two effects, one from the instrument and the other intrinsic to the star. This can be achieved 

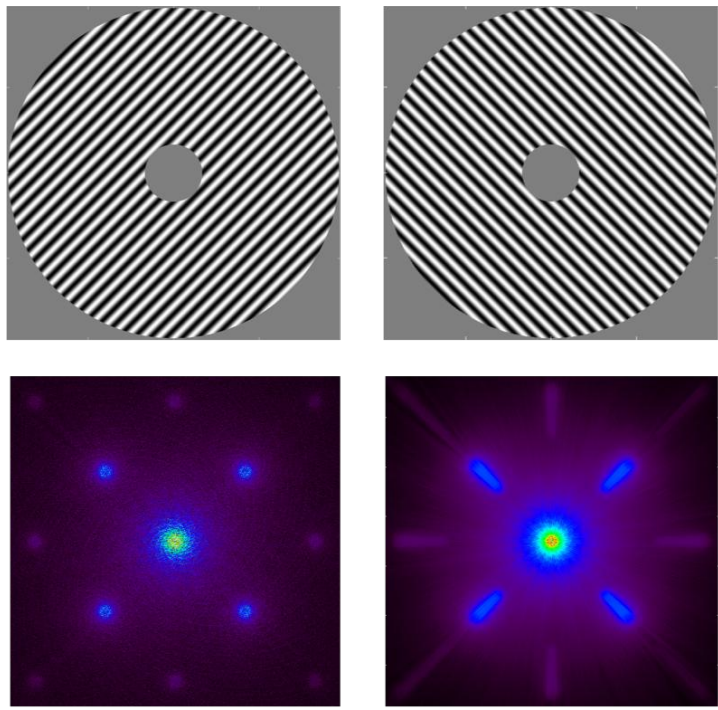

Monochromatic

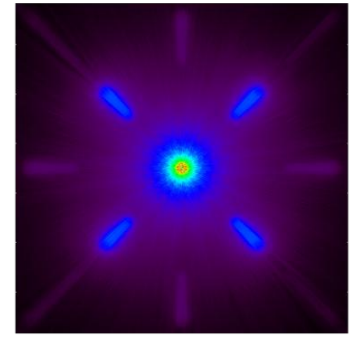

Broadband
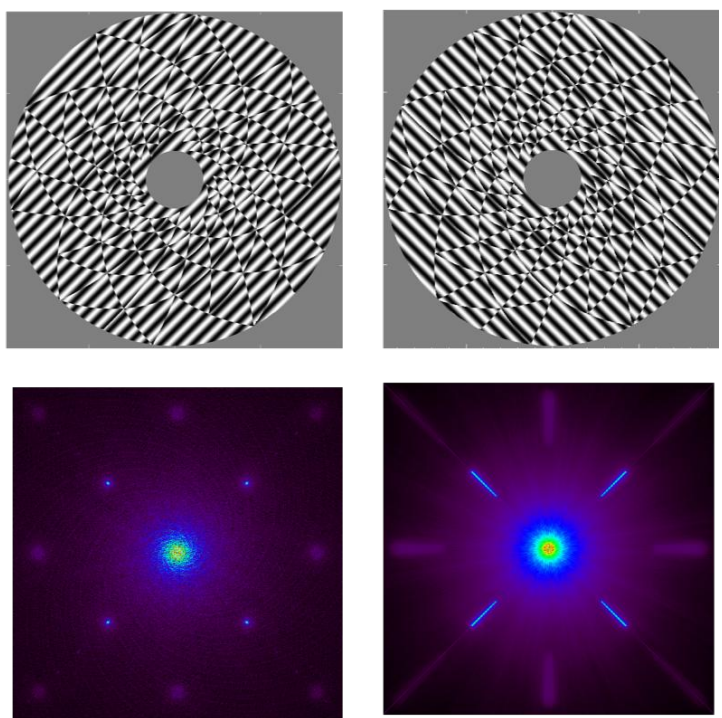

Monochromatic

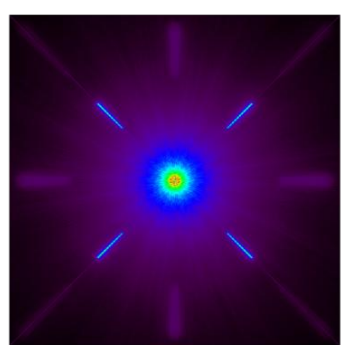

Broadband

Figure 6. Left 4 Panels: the results of imposing a simple sinusoidal grating on top of the point-design diffractive pupil given in Figure 5. Upper panels illustrate two sinusoidal phase gratings oriented orthogonally, while the Lower panels show the resulting image plane core/sidelobe features both monochromatically (left) and over a $10 \%$ fractional bandwidth (right). Right 4 Panels: as for the left set, except that the gratings now have phase flips (discussed in the text) implemented.

by simultaneously monitoring the precise stellar spectrum so that any variations can be compensated for in the data reduction.

This function is accomplished by adding a second set of diffractive features to the TOLIMAN pupil. Most simply, these could consist of a sinusoidal phase grating spanning the pupil so that the stellar spectrum can be recovered by a slitless spectrograph occupying the corners of the array detector. As for the original logharmonic spiral pattern, the development of an effective diffractive design to accomplish this task requires some sophistication. This design process is illustrated in Figure 6. The simple addition of phase gratings to the previous point-design pupil from Figure 5 results in the replication of the structured core PSF, echoed at the location of the sidelobes. As can be seen from the polychromatic simulation, this creates a very blurred slit function resulting in a challenging task to extract the spectral information encoded in the radial direction.

These considerations present a dilemmma for the optimisation of the diffractive pupil. On the one hand, the mission requires strong diffractive spread of the starlight around the PSF core to channel energy into the ruler pattern. However on the other hand, we require the grating sidelobe PSFs to be as compact and near diffraction-limited as possible so that spectral information is not compromised. Fortunately, as illustrated in the four right-hand panels Figure 6, there is a way to optimize both. This is effected by inverting the phase of the sinusiodal grating according as the primary tri-spiral switches from zero to $\pi$. As can be seen from the Figure, this is remarkably effective at simultaneously accomplishing both (at the surface of it) incompatible demands. The sidelobe PSFs are perfectly diffraction-limited Airy functions, while the central PSF maintains the complex diffractive cloud previously described.

With this hybrid "Doubly-Diffractive" pupil, we are able to provide contemporaneous spectral monitoring (with $R \sim 500$ ) of the star and so account for any subtle changes in the effective wavelength. However it is important to note that the stellar lines which are imprinted in the sidelobes also give an additional, and more direct way to ensure calibration stability. We note that the stellar lines originate from basic atomic physics, providing an absolute wavelength reference. Given the presence of such a reference, and combined with the (presumed) mechanical stability of the diffractive pupil, then it will be noted that the location of the lines in the image plane serves to fully specify the plate scale of the entire instrument. Furthermore, because our optical 
arrangement will place the stellar spectra at the corners of the detector about 2 arcminutes from the PSF core, this arrangment creates a geometrical amplification greatly augmenting the sensitivity to plate scale. Because the $\alpha$ Cen binary requires monitoring of a $\sim 5$ arcsecond binary, this method of monitoring the plate scale gives about a factor of 30 gain in angular fidelity.

Thus the doubly-diffractive pupil provides two entirely independent calibrations: both the stellar spectrum (and therefore effective wavelength) as well as the final image scale in absolute units. Of course the information provided by these two pathways is not independent; they therefore provide a way to cross-check the calibration at sub-microarcsecond scales.

\section{CONCLUSIONS AND TOLIMAN MISSION}

This paper has discussed a series of optical innovations designed to perform the required sub-microarcsecond astrometric metrology required for habitable-zone Earth mass detection. Our aim is to accomplish this with a small-aperture telescope $(<30 \mathrm{~cm})$ and therefore enabling an inexpensive and agile mission to probe this important scientific domain. In order to obtain several orbital cycles, it is expected that a minimum mission duration of $2-3$ years will be required for the final science yield.

Numerical simulations to verify the basic signal-to-noise advantages have delivered promising results, and will be reported in a forthcoming paper. Example images illustrating this process are given in Figure 7 which shows the hybrid doubly-diffractive pupil as well as the expected image-plane data when observing the $\alpha$ Centuri AB system through a narrow filter.

As the project evolves towards maturity, further enhancements and sophistication will be trialled with our suite of simulation software. In particular, the idea to also sculpt a diffractive pattern onto the secondary mirror will be explored. Placing such a structure onto all powered optics will, we believe, yield complete knowledge of the instantaneous optical configuration including the ability to constrain all relevant mechanical misalignments along the optical train. Such a scheme may be favorable for situations beyond the strict confines of astrometric science, yielding a general way to monitor telescope optical alignments.

A second promising avenue to expand the TOLIMAN science yield is to explore the potential for the diffractive pupil as a high precision photometry engine. In order to spread light over many pixels and thereby combat
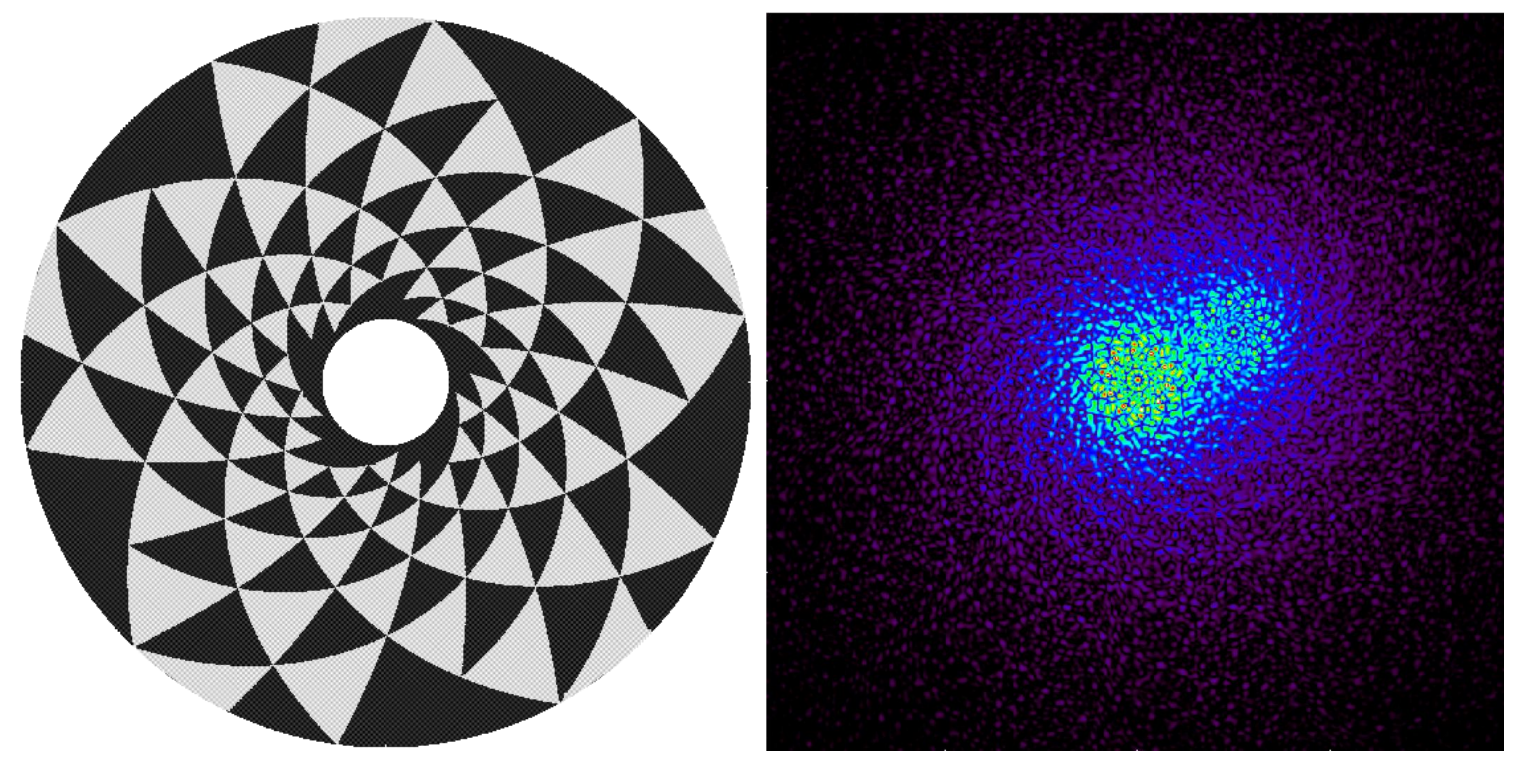

Figure 7. Left Panel: point design for the final TOLIMAN pupil depicting the tri-spiral diffractive phase steps on top of which are overlaid the weaker dual sinusiodal gratings. Right Panel: a simulated image of $\alpha$ Cen as observed in narrowband light through the TOLIMAN telescope. Substantial overlap of the diffraction patterns is fundamental to empowering the diffractive pupil methodology. 
some noise processes arising from the array such as imperfections in the flat-field, many photometric campaigns choose to deliberately degrade the PSF. This can be simply accomplished by, for example, defocussing the telescope, or in a more comprehensive way by employing diffusers. ${ }^{9}$ All such approaches satisfy the requirement to spread the light over many pixels, however they do so by creating a minimum information PSF for which it is subsequently impossible to recover high spatial resolution information. On the other hand, spreading the light by diffractive pupil techniques can easily dilute the signal over thousands of pixels, yet in principle (at least monochromatically) it preserves all information at the full diffraction limit. This means that even stars populating heavily crowded fields can be "diffused" over a wide area on the detector, yet with some simple computer analysis, yield photometric information that can be recovered at the full diffraction limit of the telescope. Such a scenario has the advantage that blended background stars will not bias photometry unless they are truly unresolved at the native resolution of the telescope.

\section{ACKNOWLEDGMENTS}

The original scientific impetus as well as critical financial and logistical support for this research program were provided by the Breakthrough Prize Foundation. The authors also acknowledge the University of Sydney who are joint partners in the study which produced the results presented.

\section{REFERENCES}

[1] "Breakthrough Initiatives: Starshot." http://breakthroughinitiatives.org/initiative/3.

[2] Shao, M., Marcy, G., Catanzarite, J. H., Edberg, S. J., Léger, A., Malbet, F., Queloz, D., Muterspaugh, M. W., Beichman, C., Fischer, D., Ford, E., Olling, R., Kulkarni, S., Unwin, S. C., and Traub, W., "Astrometric Detection of Earthlike Planets," in [astro2010: The Astronomy and Astrophysics Decadal Survey], ArXiv Astrophysics e-prints 2010 (2009).

[3] Anglada-Escudé, G., Amado, P. J., Barnes, J., Berdiñas, Z. M., Butler, R. P., Coleman, G. A. L., de La Cueva, I., Dreizler, S., Endl, M., Giesers, B., Jeffers, S. V., Jenkins, J. S., Jones, H. R. A., Kiraga, M., Kürster, M., López-González, M. J., Marvin, C. J., Morales, N., Morin, J., Nelson, R. P., Ortiz, J. L., Ofir, A., Paardekooper, S.-J., Reiners, A., Rodríguez, E., Rodríguez-López, C., Sarmiento, L. F., Strachan, J. P., Tsapras, Y., Tuomi, M., and Zechmeister, M., "A terrestrial planet candidate in a temperate orbit around Proxima Centauri," Nature 536, 437-440 (Aug. 2016).

[4] Gravity Collaboration, "First light for GRAVITY: Phase referencing optical interferometry for the Very Large Telescope Interferometer," A $\& A$ A 602, A94 (June 2017).

[5] Malbet, F., Léger, A., Anglada Escudé, G., Sozzetti, A., Spolyar, D., Labadie, L., Shao, M., Holl, B., Goullioud, R., Crouzier, A., Boehm, C., and Krone-Martins, A., "Microarcsecond astrometric observatory Theia: from dark matter to compact objects and nearby earths," in [Space Telescopes and Instrumentation 2016: Optical, Infrared, and Millimeter Wave], Proc. SPIE 9904, 99042F (July 2016).

[6] Unwin, S. C., Shao, M., Tanner, A. M., Allen, R. J., Beichman, C. A., Boboltz, D., Catanzarite, J. H., Chaboyer, B. C., Ciardi, D. R., Edberg, S. J., Fey, A. L., Fischer, D. A., Gelino, C. R., Gould, A. P., Grillmair, C., Henry, T. J., Johnston, K. V., Johnston, K. J., Jones, D. L., Kulkarni, S. R., Law, N. M., Majewski, S. R., Makarov, V. V., Marcy, G. W., Meier, D. L., Olling, R. P., Pan, X., Patterson, R. J., Pitesky, J. E., Quirrenbach, A., Shaklan, S. B., Shaya, E. J., Strigari, L. E., Tomsick, J. A., Wehrle, A. E., and Worthey, G., "Taking the Measure of the Universe: Precision Astrometry with SIM PlanetQuest," PASP 120, 38 (Jan. 2008).

[7] Chen, D., Wu, J., and Li, B., "STEP Mission: Search for Terrestrial Exo-Planets," European Planetary Science Congress 8, EPSC2013-1102 (Sept. 2013).

[8] Guyon, O., Bendek, E. A., Eisner, J. A., Angel, R., Woolf, N. J., Milster, T. D., Ammons, S. M., Shao, M., Shaklan, S., Levine, M., Nemati, B., Pitman, J., Woodruff, R. A., and Belikov, R., "High-precision Astrometry with a Diffractive Pupil Telescope," ApJS 200, 11 (June 2012).

[9] Stefansson, G., Mahadevan, S., Hebb, L., Wisniewski, J., Huehnerhoff, J., Morris, B., Halverson, S., Zhao, M., Wright, J., O'rourke, J., Knutson, H., Hawley, S., Kanodia, S., Li, Y., Hagen, L. M. Z., Liu, L. J., Beatty, T., Bender, C., Robertson, P., Dembicky, J., Gray, C., Ketzeback, W., McMillan, R., and Rudyk, T., "Toward Space-like Photometric Precision from the Ground with Beam-shaping Diffusers," ApJ 848, 9 (Oct. 2017). 